\title{
Balkanologie
}

Balkanologie Revue d'études pluridisciplinaires

Vol. VIII, $n^{\circ} 1 \mid 2004$

Volume VIII Numéro 1

\section{Pas coupables, mais responsables}

Construire la Suisse après la guerre civile (1847)

Not guilty but reponsible. Constructing Switzerland after the civil war (1847)

\section{Irène Herrmann}

\section{(2) OpenEdition}

\section{Journals}

Édition électronique

URL : http://journals.openedition.org/balkanologie/522

DOI : 10.4000/balkanologie.522

ISSN : 1965-0582

Éditeur

Association française d'études sur les Balkans (Afebalk)

\section{Édition imprimée}

Date de publication : 1 juin 2004

ISSN : 1279-7952

\section{Référence électronique}

Irène Herrmann, «Pas coupables, mais responsables », Balkanologie [En ligne], Vol. VIII, $\mathrm{n}^{\circ} 1$ | 2004, mis en ligne le 21 janvier 2010, consulté le 17 décembre 2020. URL : http://journals.openedition.org/ balkanologie/522 ; DOI : https://doi.org/10.4000/balkanologie.522

\section{(c) Tous droits réservés}




\title{
PAS COUPABLES, MAIS RESPONSABLES \\ CONSTRUIRE LA SUISSE APRÈS LA GUERRE CIVILE [184?]
}

\author{
Irène Herrmann*
}

De nos jours, la Suisse passe volontiers pour un pays paisible, peuplé de citoyens qui ne le sont pas moins. En réalité, l'image d'une contrée providentiellement épargnée par les divisions relève du cliché. Longtemps, la société helvétique a été tout aussi violente que les autres, et le calme qui semble maintenant régner dans le pays est le fruit d'efforts, de techniques conciliatoires et de structures gouvernementales appropriées, qui forment un vaste réservoir de modèles d'accommodement à méditer, voire, pour certains auteurs, à imiter ${ }^{1}$.

La plupart des politologues, soucieux de tranquillité future, ont été intéressés par le cas helvétique. Dans le cadre des réflexions théoriques qui ont accompagné le mouvement de la décolonisation, de nombreux chercheurs se sont penchés sur la pax helvetica. Les plus grands experts qui se sont alors interrogés sur les motifs de cette tranquillité étonnante l'ont attribuée à l'action conjointe de la démocratie et du fédéralisme ou à un mélange de négociation et de consensus, soit autant d'éléments résumés, à la suite de Lijphart, par la notion de démocratie consociationnelle ${ }^{2}$. Mais au-delà des spécificités institutionnelles du pays, ces politologues se sont accordés à considérer que les conditions préalables et nécessaires de l'efficacité de la conflict resolution

\footnotetext{
•FNRS/Université de Genève. (irene.herrmann@lettres.unige.ch)

${ }^{1}$ Cette réputation est aussi largement entretenue " de l'intérieur $n$. Voir, par exemple, Linder (Wolf), Swiss Democracy. Possible solutions to Conflict in Multicultural Societies, New York : St Martin Press, 1994, ou Steiner (Jürgen), " Power Sharing : Another Swiss Export Product ? ", in Montville (Joseph V.), ed., Conflict and Peacemaking in Multiethnic Societies, Lexington : Lexington Books, 1989 ; ou, de manière plus nuancée, Schoch (Bruno), Switzerland - A Model for Solving Nationality Conflicts ?, Frankfurt : PRIF, 2000.
}

${ }^{2}$ Lijphart (Arendt), " Typologies of Democratic Systems ", Comparative Political Studies, 1 (1), 1968. 
suisse s'articulent autour de deux facteurs fondamentaux : à savoir la prospérité économique de la Confédération et, surtout, an older tradition of elite accomodation ${ }^{3}$.

Bizarrement, on constate que les premiers concernés par cette interprétation des choses, à savoir les historiens, ne se sont guère sentis interpellés par cette question. En fait, ils ont préféré détailler les dissensions qui ont menacé l'existence puis le maintien de l'entité suisse 4 . L'une de celles qui a le plus attiré les regards, ces dernières années, est la guerre civile du Sonderbund qui divisa le pays, en 1847. Mais, en dépit de l'engouement que le sujet a suscité, les chercheurs n'ont guère analysé la manière dont ce conflit s'était achevé, sur le court et le moyen terme 5 . Or, la fin de cet affrontement constitue un terrain d'investigation idéal pour décortiquer la fameuse "tradition d'accommodement " suisse ; c'est-à-dire pour appréhender ses composantes et ses mécanismes, afin de voir, en dernière analyse, si cette tradition est aussi suisse, aussi ancienne et, finalement, aussi efficace ou exemplaire que cela.

\section{LE SONDERBUND}

La réputation de calme qui caractérise la Suisse est d'autant plus étonnante que durant le Moyen Âge, les Helvètes étaient réputés pour leur cruauté6. Soldats redoutés dans l'Europe entière, ils cessent d'œuvrer sur la scène internationale au début des Temps modernes. À l'abri de la neutralité acquise au lendemain de la bataille de Marignan, ils se consacrent entièrement à leurs luttes intestines. À l'instar de l'ensemble du vieux continent, les Suisses se déchirent alors entre catholiques et protestants, riches et pauvres puis, au lendemain de

\footnotetext{
3 Cité par McRae (Kenneth D.), "Theories of Power-Sharing and Conflict Management ", in Montville (Joseph V.), ed., op.cit, p. 95.

${ }^{4}$ Bois (Pierre du), Union et division des Suisses. Les relations entre Alémaniques, Romands et Tessinois aux XIXe et XXe siècles, Lausanne : éd. de l'Aire, 1983 ; ainsi que, plus récemment, "La Suisse, pays du consensus ? ", Traverse, 8 (3), 2001.

5 Ce conflit a donné lieu à la monographie capitale de Bucher (Erwin), Die Geschichte des Sonderbundskrieges, Zurich : Berichtshaus, 1966 et, ces dernières années, à quelques articles qui plaident pour en renouveler l'approche. Voir, Moos (Carlo), "Fragen an den Sonderbund ", Der Geschichtsfreund. Mitteilungen des Historischen Vereins der fünf Orte Luzern, Uri, Unterwalden ob und nid dem Wald und Zug, (149), 1996 ; ou Jorio (Marco), " "Gott mit uns." Der Bund des Sonderbundes mit Gott ", in Ernst (Andreas), Tanner (Albert), Weishaupt (Matthias), Hg., Revolution und Innovation. Die Konfliktreiche Entstehung des schweizerischen Bundesstaaten von 1848, Zurich, 1998. En ce qui concerne les rares titres étrangers, il convient de citer celui de Remak (Joachim), A Very Civil War : the Swiss Sonderbund War of 1847, San Francisco : Westview Press, 1993.
}

${ }^{6}$ Sieber-Lehmann (Claudius), Spätmittelalterischer Nationalismus. Die Burgunderkriege am Oberrhein und in der Eidgenossenschaft, Göttingen : Vandenhoeck \& Ruprecht, 1995, pp. 204 et sq. 
la Révolution française, entre progressistes et conservateurs. Bon gré mal gré, ces différents clivages sont régulés par une institution faîtière, la Diète. Et quand celle-ci est incapable de remplir son office de pacification, ce qui arrive souvent, les Suisses usent alors de pratiques largement éprouvées par la tradition, telles que l'arbitrage, les compensations financières ou les conférences de conciliation?.

Au milieu du $\mathrm{XIX}^{\mathrm{e}}$ siècle, toutes ces techniques se révèlent impropres à juguler un antagonisme majeur, cumulant les divers facteurs de division qui étaient apparus au cours des siècles précédents. Au lendemain des révolutions de 1830 , la contrée tend à se diviser lentement en deux camps antagoniques. D'un côté, se regroupent des cantons ruraux, catholiques et désireux de conserver le statu quo ante. Ils sont situés au centre du pays, soit à l'endroit que l'on considère comme étant le berceau historique de la Confédération helvétique. En périphérie, on trouve des cantons plus industrialisés, majoritairement protestants et radicaux, désirant une meilleure cohésion politico-économique de la contrée. Au début des années 1840, les relations entre ces deux ensembles se détériorent. Les incidents se multiplient en un engrenage hostile où les arguments confessionnels et politiques se superposent, introduisant un climat de défiance fondamentale ${ }^{8}$. Le développement de ce cercle vicieux est favorisé par la certitude qu'ont les uns d'être menacés dans leur pratique religieuse, ce qui les incite à se replier sur un fédéralisme outrancier, seule garantie de leur souveraineté cantonale. Les autres interprètent cette attitude comme une preuve d'ultramontanisme et, surtout, comme un obstacle à leur ambition de centraliser la Confédération, voire d'en faire un véritable État-nation.

Pour défendre leurs intérêts qu'ils estiment menacés, sept cantons catholiques, à savoir Uri, Schwyz, Unterwald, Zoug, Valais, Fribourg et Lucerne s'unissent en une ligue séparée dite, en allemand, Sonderbund. À l'annonce de cette alliance, tous les autres petits États helvétiques se coalisent à leur tour et, insensiblement, chacun se prépare à la guerre. Au début du mois de novembre 1847, le conflit éclate. Il est remporté par le camp radical qui s'impose en quelques trois semaines seulement, et en faisant moins d'une centaine de morts 9 . Cette célérité est facilitée par la négligence militaire des chefs du parti catholique, par leur incapacité à obtenir de l'aide de l'étranger, comme ils en

7 Würgler (Andreas), « Aushandeln statt protestieren. Zur Konfliktkultur der alten Eidgenossenschaft im Vergleich mit Frankreich und mit dem Deutschen Reich (1500-1800) ", Traverse, 8 (3), 2001 et Maissen (Thomas), " Disputatio de Helvetiis an natura consentiant ", Traverse, 8 (3), 2001.

${ }^{8}$ Ernst (Andreas), "Charisma, Gemeinschaft und Konflikt in der Gründungsphase des Bundesstaates, 1830-1848 n, in Ernst (Andreas), Tanner (Albert), Weishaupt (Matthias), Hg., op.cit.

9 Soit un décès pour 24000 habitants, alors que la Guerre civile américaine fit un mort pour quinze personnes. 
avaient le dessein, et par l'impréparation évidente de leurs troupes. Par ailleurs, cette vitesse de mouvement est délibérément orchestrée par leurs adversaires qui bénéficient, pour ce faire, d'un meilleur armement et d'un chef remarquable, le général Dufour, d'ailleurs formé au sein de système napoléonien $^{10}$. Mais surtout, cette rapidité s'insère parmi une large panoplie de moyens destinés à rendre le conflit peu meurtrier, en vue d'une reconstruction aisée de la cohésion nationale perdue.

Car, dès le début du conflit, les futurs vainqueurs avaient été préoccupés de transformer cette contrée divisée, meurtrie et entourée de pays hostiles en un véritable État-nation, uni, cohérent, solidaire et susceptible de se repositionner sur la carte de l'Europe. Il s'agissait d'éviter le cercle vicieux de la vengeance et des représailles afin de proposer un projet de société commun à tous les Suisses. Les solutions qu'ils apportent à ce vaste problème sont alors autant de variations sur le thème de la responsabilité, au sens de Hans Jonas, " en vertu duquel je me sens responsable non en premier lieu de mon comportement et de ses conséquences, mais de la chose qui revendique mon agir ${ }^{11}$ "; elles se déclinent sous la forme de la non-responsabilité, de la déresponsabilisation et de la " surresponsabilisation".

\section{LA NON-RESPONSABILITÉ}

Un certain nombre de facteurs d'apaisement sont totalement indépendants de la volonté tant des vainqueurs que des vaincus ; les protagonistes du conflit n'étant en l'occurrence absolument pas responsables des effets positifs que ces phénomènes purent avoir.

Le premier se présente comme un hasard chronologique favorable. Il se trouve, en effet, que les petits cantons du Sonderbund avaient pris contact avec l'étranger, dans l'espoir que les grandes puissances catholiques avoisinantes, telles que la France ou l'Autriche, leur procureraient des hommes et des armes. Ces deux monarchies étaient d'ailleurs favorables à un soutien de ce genre, dans la mesure où il ouvrait des perspectives annexionnistes tout à fait intéressantes. Mais l'une et l'autre furent prises de court par la rapidité de la

\footnotetext{
${ }^{10}$ Langendorf (Jean-Jacques), éd., "Aimez-moi comme je vous aime ". 190 lettres de G. H. Dufour à A. Pictet, Vienne : Karolinger, 1987.
}

11 Jonas (Hans), Le principe responsabilité. Une éthique pour la civilisation technologique, Paris : Flammarion, 1990, p. 132. 
guerre civile helvétique ${ }^{12}$ et, plus encore, par l'éclatement de multiples révolutions au début de l'année 1848 , qui ajournèrent indéfiniment leur projet d'intervention en Suisse.

Le deuxième facteur de conciliation se présente comme un hasard géographique favorable. On constate aisément que l'essentiel des cantons sécessionnistes se situent au cœur même de la Confédération. Il était donc difficile pour eux de recevoir de l'aide extérieure mais aussi de se séparer du reste des Suisses ${ }^{13}$.

Le troisième élément est d'ordre économique. Même si le pays était encore, au milieu du XIX ${ }^{\mathrm{e}}$ siècle, une contrée pauvre, du moins existait-il entre les cantons urbains, proto-industrialisés, protestants, et les cantons ruraux, catholiques une grande complémentarité industrielle, doublée d'une tradition d'échanges commerciaux dont la poursuite était de l'intérêt de tous, des vainqueurs comme des perdants ${ }^{14}$.

Enfin, à tout cela s'ajoute un élément structurel, de nature confessionnelle. Si les petits cantons se sont ligués entre eux pour défendre leur religion, cette même religion leur enseignait un fatalisme et une obéissance qui se prêtaient mal aux mouvements de guérilla. Autant les populations du centre de l'Helvétie pensaient se battre avec Dieu et pour Dieu ; autant, une fois défaites, elles ont l'impression d'avoir été abandonnées par Lui. Dès lors, elles jugent que leur cause n'était pas aussi sacrée qu'elles le pensaient ou, pire encore, que la victoire des radicaux est une punition divine contre laquelle il est vain de se révolter ${ }^{15}$. Les vainqueurs sont alors conscients de l'état d'esprit qui règne chez leurs adversaires et n'hésitent pas à en tirer profit pour élaborer leurs propres techniques conciliatoires, à commencer par une vaste entreprise de " déresponsabilisation ".

\footnotetext{
${ }^{12}$ En fait, la France, la Grande Bretagne, la Prusse, la Russie et l'Autriche n'expédièrent leur offre (comminatoire) de médiation que le 30 novembre, soit un jour après la cessation des hostilités. Ce truculent contretemps permit aux vainqueurs du Sonderbund une lettre emprunte d'ironie déférente, saluant poliment la tardive sollicitude manifestée par les Puissances. Dans cette réponse, ils réduisaient le conflit à un simple déploiement d'armes destiné à " faire rentrer une faction rebelle dans le devoir, rétablir l'ordre et la tranquillitẻ [et] maintenir la sûreté intérieure de la Suisse " (Archives d'État de Genève [désormais AEG], Annexes des Registres du Conseil d'État [RC Ann] AF 1847 II, (230) : Lettre de la Commission de la Diète à son Excellence $M$. le Ministre des affaires étrangères à Paris, du 6 décembre $1847, \mathrm{f}^{\circ} 1$ ).
}

${ }^{13}$ Le Québec s'est d'ailleurs heurté à ce type de problèmes dans ses tentatives souverainistes.

${ }^{14}$ Cette thèse sous-tend l'ensemble de l'ouvrage de Bergiex (Jean-François), Histoire économique de la Suisse, Lausanne : Payot, 1988.

${ }^{15}$ Moos (Carlo), " Dimensionen eines Bürgerkrieges. Für eine Neubewertung des Geschehens um den Sonderbund ", in Studer (Brigitte), Hg., Etappen des Bundesstaates. Staats- und Natiomnbildung der Schweiz, 1848-1998, Zurich, 1998, pp. 22 et sq. 


\section{DÉRESPONSABILISER}

Plus que la non-responsabilité, dont les effets échappaient grandement au contrôle des contemporains, la déresponsabilisation s'intègre dans les moyens mis en œuvre par les vainqueurs pour transformer la Suisse en État-nation, selon les normes géopolitiques en vogue à l'époque. La Suisse ainsi créée ou recréée devait être gérée par des préceptes progressistes, admettant le suffrage universel masculin, une plus grande concentration administrative et une liberté économique accrue ${ }^{16}$.

Ce projet ambitieux impliquait une vaste réflexion sur les moyens susceptibles de le rendre possible, sachant que les nouveaux dirigeants helvétiques n'avaient pas l'argent nécessaire pour soutenir une longue guérilla, ni même le temps de le faire, puisqu'ils étaient entourés de Grandes Puissances aux appétits territoriaux pour le moins menaçants. Dans ce contexte, interviennent plusieurs résolutions qui contribueront, en effet, à une pacification immédiate de la Suisse. Tout d'abord, l'orchestration de cette fameuse rapidité, certes favorisée par l'incurie du camp sonderbundien, mais délibérément accentuée pour éviter un maximum de morts. Ensuite, d'innombrables injonctions au calme, au respect des propriétés et surtout des personnes ${ }^{17}$.

En principe, ces appels auraient pu rester lettre morte. Les officiers du camp fédéral auront pourtant l'intelligence de les entourer d'une rhétorique de "déresponsabilisation " du camp adverse. Tout au long du conflit, on explique aux soldats qu'ils ne doivent pas s'en prendre à leurs ennemis, ni civils ni même militaires. Car ces ennemis seraient des frères, des Suisses et, surtout, ils ne seraient pas responsables des hostilités en cours. Bien mieux, ils auraient été l'objet d'un complot, trompés dans leurs croyances et leurs représentations afin de les contraindre à se battre contre leurs propres concitoyens, alors même que leur plus cher désir aurait été de vivre en paix ${ }^{18}$.

\footnotetext{
${ }^{16}$ Sur cet événement, ses causes et ses conséquences, voir : Hildbrand (Thomas), Tanner (Albert), Hg., Im Zeichen der Revolution. Der Weg zum schweizerischen Bundesstaat 1798-1848, Zurich, 1997 ; Kästli (Tobias), CH - eine Republik in Europa. Der Schweizerische Nationalstaat seit 1798, Zurich, 1998 ; Studer (Brigitte), Hg., op.cit.

${ }^{17}$ Le colonel fédéral Rilliet, pénétrant le 29 novembre 1847 dans le Valais sonderbundien, harangua ainsi ses hommes: " Soldats confédérés de la 1 ère division (...) Que l'occupation du Valais reste pure de tout excès et de toute violence et que chacun de vous en rentrant bientôt dans ses foyers, y rentre la conscience pure et puisse sans rougir serrer les mains à ses confédérés du Valais, qu'au prix de tant de sacrifices vous avez rendu à la liberté et au bonheur " [AEG, RC Ann 1847 II AF (188)]. Par la suite, on veillera à ce que les objets pillés en dépit des interdictions réitérées, soient restitués à leurs propriétaires.
}

${ }^{18}$ Les habitants des cantons sécessionnistes sont envisagés comme des victimes ; manipulées par " ces gens qui, sous le prétexte d'assurer à leur canton l'indépendance vis-à-vis de la Confédération, et de 
À ce stade de l'argumentation intervient un processus d'accusation à l'encontre de ceux qui sont censés avoir fomenté cette ignoble tromperie et entraîné les catholiques conservateurs tout comme les progressistes protestants dans la guerre civile. Les coupables ainsi désignés se recrutent dans deux cercles distincts. Les premiers sont les Jésuites. Venus de l'étranger et porteurs d'une idéologie teintée d'ultramontanisme, ils sont accusés d'avoir voulu engager la Suisse dans une voie supra-nationale, supervisée par le Vatican, qui n'était pas la sienne. Le second groupe incriminé est le patriciat des petits cantons du Sonderbund : il a, précisément, soutenu les projets meurtriers de la Compagnie de Jésus et exercé une politique qui ne servait que ses intérêts propres.

Ces deux coupables génériques servent de véritables boucs émissaires au conflit, en un processus lourd de conséquences pratiques et idéologiques. Les Jésuites sont immédiatement chassés de Suisse et les membres des familles aristocratiques du Sonderbund sont soumis à diverses peines prévoyant l'emprisonnement avec chaînes des principaux leaders - qui préféreront tous fuir - et de lourdes amendes pour les autres. Mais la portée principale de ces mesures d'ostracisme est d'ordre symbolique. Par ricochet, cette opération blanchit tout le reste de la population, soit l'immense majorité des Helvètes, qui sont présentés comme des victimes innocentes n'ayant jamais cherché à se combattre mutuellement. Par ricochet, toujours, ces boucs émissaires indiquent aux citoyens qui ils sont et dans quelle direction doit se diriger le nouveau pays. Par opposition aux Jésuites, les Suisses sont censés vouloir faire (re)naître un pays indépendant, dégagé de la tutelle étrangère ; et par opposition aux aristocraties locales, éminemment conservatrices, les confédérés nouveaux se doivent d'être progressistes.

En faisant porter l'entière responsabilité du conflit à un nombre très réduit de personnes et, partant, en déresponsabilisant toutes les autres, les vainqueurs savaient pouvoir s'assurer un pays tranquille, du moins dans l'immédiat. Afin de consolider l'avenir de leur nouvelle construction nationale, ils auront bientôt recours au procédé inverse, soit à une responsabilisation exacerbée, la " surresponsabilisation ".

maintenir l'existence cantonale qui n'était menacée par personne, livrent l'instruction publique et l'éducation du peuple à des hommes soumis à un pouvoir étranger. [Il est indispensable de repousser] les idées de ces hommes aveuglés, de ces hommes insensés, qui voulaient arrêter la marche du progrès et faire reculer la civilisation. Car si le Sonderbund eût été victorieux, la Suisse était destinée à servir de boulevard à la réaction européenne et à la restauration de toutes les aristocraties n (AEG, RC Ann 1848 II, AF, (39), " Discours adressé au Grand Conseil Constituant du Valais par M. le Conseiller d'État Delarageaz, le 28 décembre $1847 n$ ). 


\section{«SURRESPONSABILISER »}

Les quelques décennies qui succèdent au Sonderbund sont consacrées au façonnement de la première et seule république d'Europe. Cette entreprise nécessite de cimenter le substrat social et national sur lequel on la bâtit. Il est ainsi vital d'éviter une nouvelle guerre civile qui puiserait sa source dans les souvenirs de l'ancienne. Dans ce cadre, les élites du pays élaborent un système politique qui, sous prétexte d'intégrer les minorités linguistiques ou religieuses dans le processus de prise de décision politique, muselle en réalité toutes leurs revendications ${ }^{19}$. Parallèlement, les gouvernements helvétiques successifs, néanmoins conscients des risques inhérents aux droits populaires octroyés à leurs concitoyens, tentent par différents procédés de les rendre raisonnables dans l'emploi des moyens démocratiques mis à leur disposition et, plus encore, de les rendre responsables.

À cet effet, l'une des méthodes les plus couramment employées est le recours systématique à l'histoire. Mais au lendemain de la guerre civile, l'histoire ainsi convoquée se conçoit comme une instance de jugement supérieure à l'aune de laquelle s'évalue toute action humaine et, de ce fait, ne s'apparente guère au classique récit des faits écoulés ${ }^{20}$. On note ainsi l'étonnante rareté des références au conflit, qui s'explique par la proximité temporelle du Sonderbund et le désir de ne pas ouvrir des cicatrices encore fraîches. On observe également la surprenante occultation des héros mythiques de la Suisse qui, quant à elle, se comprend dans un contexte de concurrence rhétorique. De fait, l'évocation des Trois Suisses était une figure obligée du discours conservateur et se prêtait donc mal à la récupération. En outre, elle avait été popularisée par la République helvétique ; soit le régime révolutionnaire de sinistre mémoire qui, entre 1798 et 1803 , avait pris exemple sur le modèle fran-

19 Gruner (Erich), Die Wahlen in den schweizerischen Nationalrat 1848-1919, Berne, 1978, tome III, ainsi que l'analyse à la fois très complète et pointue de Tanner (Albert), " Ein Staat nur für die Hablichen ? Demokratie und politische Elite im frühen Bundesstaat n, in Studer (Brigitte), Hg., op.cit., pp. 63 et sq. Ces deux textes montrent, entre autres, la manière subtile dont les radicaux vainqueurs du Sonderbund exploitèrent les possibilités offertes par le système majoritaire, de façon à ce que leur position dominante sur l'èchiquier politique suisse ne puisse en aucun cas être remis en question.

${ }^{20}$ Ce phénomène s'observe avec netteté durant les périodes de crises, où le nouvel édifice helvétique semble mis en danger. Au lendemain de l'alerte de Neuchâtel, après qu'un coup d'État royaliste eut vainement tenté de remettre le roi de Prusse à la tête de ce canton au statut longtemps hybride, le Conseil fédéral félicite les troupes suisses en termes significatifs : "Soldats citoyens ! Gardez dans votre souvenir ces journées d'une époque émouvante (...), ces journées dans lesquelles la Suisse a donné un si bel exemple d'union et de fraternité (...). Défenseurs de la patrie, votre fidélité, votre dévouement, votre abnégation ne peuvent jamais tomber dans l'oubli, la postérité reconnaissante vous élèvera un monument impérissable et bien mérité, et l'histoire de la Nation vous accordera une large place dans ses annales n (Feuille fédérale, Berne, 1857 , t. I, pp. 70-71; c'est nous qui soulignons). 
çais $^{21}$. Enfin, la référence aux valeureux pâtres de la saga helvétique posait un problème " géo-historique " : tous ses personnages étaient issus du berceau mythique de la Confédération, certes, mais également de ce qui avait fait le cœur du Sonderbund.

Quelques générations après la fin du Sonderbund, ces inconvénients s'effacent d'eux-mêmes, voire se transforment en avantages. Ainsi, dès la fin de la guerre franco-prussienne de 1870 , le temps commence à faire son effet. Les témoins directs du Sonderbund ainsi que la vieille garde historienne ont disparu. Mieux encore, ils laissent place à de jeunes savants formés dans les universités allemandes. Ces chercheurs sont de fervents partisans de la nouvelle Confédération de 1848 dont la création constitue, selon eux, l'aboutissement naturel du cheminement helvétique ${ }^{22}$. Ils réinventent un récit de la nation qui intègre tant les fables des origines que l'épisode mouvementé de 1847, en une narration sur laquelle le gouvernement helvétique vient rapidement s'appuyer.

Après le Kulturkampf (dont la phase la plus aiguë se termine en Suisse vers 1876), les autorités fédérales pensent en effet (re)colmater le pays en utilisant la vieille histoire mythique et, surtout, son ancrage géographique. Dès la fin du XIX $\mathrm{XI}^{\mathrm{e}}$ siècle, les magistrats n'hésitent plus à célébrer les héros légendaires de la Confédération; de sorte que la première fête nationale, le $1^{\mathrm{er}}$ août 1891 , a lieu au bord du lac des Quatre Cantons ${ }^{23}$. Le lieu choisi est celui où Guillaume Tell est censé avoir tiré sur la pomme qui ornait la tête de son fils ; mais également celui où, quelques 50 années auparavant, les dirigeants catholiques avaient décidés de faire sécession et avaient ainsi enclenché le processus menant à la guerre civile.

Le message diffusé par l'histoire est simple. Il s'agit, tout d'abord, de revendiquer l'héritage des pâtres mythiques en présentant les réalisations de l'État fédéral et leur évidente excellence comme une émanation des luttes d'indépendance menées au XIII ${ }^{\mathrm{e}}$ siècle déjà. Le but de ce discours est double. D'une part, il ambitionne de réintégrer pleinement les habitants des petits cantons dans l'existence même de la Confédération en soulignant que ses concepteurs,

\footnotetext{
${ }^{21}$ Schenk (Chr.), Das Geschichtsbild der Helvetiker : helvetische Politiker als Geschichtsschreiber : Peter Ochs, Philipp Albert Stapfer und Frédéric-César de Laharpe, Fribourg : mémoire de licence, 1998.
}

${ }^{22}$ Buchbinder (S.), Der Wille zur Geschichte. Schweizergeschichte um 1900 - die Werke von Wilhelm Oechsli, Johannes Dierauer und Karl Dändliker, Zurich, 2002; Walter (F.), « L'historiographie suisse depuis le XVIIIe siècle n, Dictionnaire historique de la Suisse, à paraître (article gracieusement communiqué par l'auteur que nous remercions ici).

${ }^{23}$ Zimmer (Oliver), "Competing memories of the nation : liberal historians and the reconstruction of the Swiss past 1870-1900 ", Past and Present. A Journal of Historical Studies, (168), August 2000 ; Zimmer (Oliver), A Contested Nation. History, Memory and Nationalism in Switzerland, 1761-1891, Cambridge/New York : Cambridge University Press, 2003, pp. 189 et sq.; Kreis (Georg), « Der zweite Gründungsmythos der Eidgenossenschaft. Zur Entstehung des Nationalfeiertages von 1291 n, Mitteilungen des Historischen Vereins des Kanton Schwyz, 82, 1990, pp. 159-200. 
soit Guillaume Tell, Winkelried et les autres, sont issus de leurs contrées qui, à ce titre, doivent être considérées comme les plus suisses de la Suisse ! Par ailleurs et surtout, il s'agit de tirer le meilleur parti possible de l'origine non plus géographique mais sociale des héros de la saga suisse. En effet, ils sont tous issus du tiers-état. De ce fait, tout Confédéré contemporain pouvait légitimement se penser le descendant de si illustres ancêtres.

Ce sentiment de parenté abusif, joint à l'idée que la Suisse serait devenue parfaite grâce à l'action de ses valeureux fondateurs médiévaux, conduit presque logiquement à l'adoption de postures complémentaires. L'une est la fierté de vivre dans un pays aussi beau grâce à des actions moyenâgeuses aussi héroïques ; l'autre est la peur de ruiner l'œuvre si merveilleuse des ancêtres. Ce mélange débouche sur une tendance générale au calme et à l'obéissance. Souvent même, les mots d'ordre du gouvernement ne sont pas simplement respectés mais devancés. Ainsi, en dépit de leurs luttes farouches contre la bourgeoisie, les ouvriers acceptent, au début des hostilités de 1914, de remettre la totalité du pouvoir fédéral aux mains de l'exécutif national, voire de revenir sur des acquis sociaux pourtant difficilement soutirés. Une abnégation similaire s'observe à la veille de la Seconde Guerre mondiale, quand les syndicats s'engagent à conclure avec les milieux patronaux une " paix du travail ", signant ainsi une trêve illimitée de leurs actions de débrayage qui imposait le règlement amiable de tous les conflits présents ou à venir. À l'évidence, la bonne volonté de la population la moins aisée de Suisse est ici dictée par les circonstances extérieures et l'intime conviction des préjudices que causerait la guerre si les dirigeants helvétiques ne se montraient pas assez forts pour pouvoir imposer la neutralité du pays.

Cette propension au renoncement ne se manifeste pas seulement en période de menace internationale. Dans bien des cas, le peuple suisse a refusé des mesures pourtant destinées à lui simplifier l'existence et proposées par les sphères gouvernementales elles-mêmes. Le retard notable de la Confédération helvétique dans le domaine des assurances maladie et accident n'a pas été délibérément entretenu par une classe dirigeante plus soucieuse de ses propres intérêts que du bien-être de ses administrés. Bien au contraire, ce sont ces derniers qui, à la faveur des pouvoirs étendus que leur conférait la démocratie, ont régulièrement rejeté les initiatives officielles destinées à améliorer la couverture sociale des citoyens suisses. Dans le même ordre d'idées, on note que l'électorat helvétique repousse couramment les suggestions de baisses d'impôts ou les réductions du temps de travail ${ }^{24}$. En fait, l'essentiel de la vie politique suisse est marquée, sinon freinée, par une logique éloquemment épinglée par le politologue Daniel-Louis Seiler : " le Conseil fédéral [soit l'exécutif

${ }^{24}$ Pour le résultat détaillé des " votations " suisses, voir : http://:www. admin.ch/ch/f/pore/va/index/html 
national], dit-il, propose des législations progressistes que l'Assemblée fédérale [le parlement] amende dans un sens plus conservateur, pour les voir souvent rejetées par le peuple souverain qui leur trouve un parfum trop gauchiste $n^{25}$. Le plus curieux, dans ce processus hautement handicapant, est que les objets ainsi présentés et rejetés sont souvent destinés à soulager la population, au détriment des caisses de la Confédération. Tout se passe donc comme si le ressortissant suisse estimait qu'il devait endosser lui-même les responsabilités qu'on délègue habituellement à l'État ; comme si le fait de vivre en démocratie prenait un sens holiste où le citoyen devait se montrer prêt à assumer lui-même la part d'autorité que les instances gouvernementales n'exerceraient pas sur lui ; ou, mieux encore, comme si la prospérité de la res publica le concernait personnellement, à titre privé.

Le résultat ultime de cette campagne de sensibilisation citoyenne par le passé aboutit ainsi à une surresponsabilisation citoyenne. Et si cette dernière engendre une évidente propension au calme, elle connaît aussi deux dérives néfastes. Sur le plan personnel, elle est facilement autodestructrice ${ }^{26}$ et à un niveau beaucoup plus général, elle mène à l'immobilisme politique qui caractérise l'essentiel du XX ${ }^{\mathrm{e}}$ siècle helvétique ${ }^{27}$.

\section{CONCLUSION}

En fin de compte, la Suisse est ainsi sortie de la violence sans trop de souffrances et sans trop de risques de récidive sérieuse. Ce calme n'est pas le fruit d'une vielle tradition d'accommodement comme l'ont si souvent prétendu les politologues. Il est le produit de hasards qui n'ont pas vraiment poussé les Helvètes à bien s'entendre entre eux mais ne leur ont pas non plus donné l'envie de vivre avec d'autres. Il est également l'aboutissement de techniques institutionnelles ou idéologiques que l'on n'hésiterait pas, aujourd'hui, à qualifier de " politically uncorrect ", soit d'une part le subtil musellement des minorités et, d'autre part, la désignation très claire de boucs émissaires nationaux. Il est enfin le résultat d'une conscience exacerbée de soi, d'une

\footnotetext{
25 Seiler (Daniel-Louis), "La Suisse comme Démocratie consociative : essai de destruction d'un mythe de science politique n, Passé pluriel. En hommage au professeur Roland Ruffieux, Fribourg : Éditions Universitaires, 1991, p. 346.

${ }^{26}$ Jost (Hans-Ulrich), « Critique historique du consensus helvétique. Si le légendaire Guillaume Tell et le mythe du serment du Grütli ne nourrissent plus guère l'imaginaire de la jeune génération, la Suisse comme pays modèle du consensus est un cliché qui se porte bien n, Traverse, 8 (3), 2001, pp. 57-79.

${ }^{27}$ Ce reproche est, semble-t-il, encore d'actualitè. À cet égard, voir notamment les réactions de la presse à l'annonce (le 5 mai 2004) de la régression de la Suisse dans le classement en matière de compétitivité mondiale qui, toutes, soulignent, l'influence néfaste de l'inertie politique helvétique sur le développement économique du pays.
} 
surresponsabilisation citoyenne qui conduit tout un chacun à exercer sur luimême la part d'autorité que l'État n'assume pas.

En dépit de son efficacité, on peut se demander si un tel modèle est imitable. Tout d'abord, il doit l'essentiel de son efficience au facteur temps. Les Suisses ont eu tout loisir de panser puis d'oublier leurs blessures. Mieux encore, ils ont bénéficié de la lenteur du développement démocratique qui, tout en leur permettant d'aller voter, ne laissait à personne la possibilité de croire qu'il avait un droit imprescriptible au bonheur.

À cet égard, l'exemple helvétique plaide d'ailleurs moins pour l'utilisation de techniques conciliatoires précises que pour l'adaptation des moyens de pacification aux contingences du lieu, du moment ou de la tradition historique. En outre et pour conclure, sachant, surtout, que cette opération peut mener à un attentisme potentiellement destructeur, on peut même se demander si l'imitation d'un tel modèle ne serait pas nuisible. 